ARTICLE

Received 8 Oct 2014 | Accepted 30 Apr 2015 | Published 9 Jun 2015

DOI: $10.1057 /$ palcomms.2015.12 OPEN

\title{
A deterministic model of a research organization's evolution and dynamics of performance
}

Prashant Goswami ${ }^{1}$, Shiv Narayan Nishad ${ }^{1}$ and Dhanya Selvaratnam ${ }^{1}$

\begin{abstract}
Quantitative descriptions of complex social systems hold promise for many applications such as understanding and quantifying group behaviour, organizational performance and inter-personal interactions. Since social systems are interacting and evolving systems, dynamical modelling of them enables the possibility to study time evolution under different scenarios in a quantitative and possibly predictive framework. There are, however, several challenges in developing such dynamical models, one of which is that unlike in physical systems, it is difficult to identify unambiguous, let alone unique, casual relations in social dynamics. A further major difficulty is in quantifying attributes like performance, personal choice and leadership. Here, we provide such a quantitative model of a sociological system, namely a research organization, with its performance as a dynamical variable. We use the model to study the evolution and sensitivity of the performance of a research organization under different conditions. The performance is measured as the sum of contributions from the individual members of the organization in terms of metrics, such as number of research publications. The individual performances are driven by various benchmarks, personal goals and other processes that respond to time-dependent internal and external factors. The factors that arise from institutional and individual aspects, like institutional average and national benchmark, are represented mathematically to describe the dynamics. The model demonstrates complex behaviour that a research institution can exhibit in response to internal as well as external factors. The model is applied to quantify the roles of various processes like initial selection criteria and leadership response in the institutional dynamics and the categories of performers. The novel feature in our formalism is a somewhat mechanistic, and deterministic, description of a research organization's evolution over time. Our results demonstrate that a social system such as a research organization can be modelled as an initial and boundary value dynamical system. Unlike qualitative or static models, such a dynamical model allows us to chart institutional trajectories under different organizational conditions. This concept and the methodology can be extended to other social systems-such as electorates or a publicly funded organization-with appropriate dynamical variables.
\end{abstract}

\footnotetext{
${ }^{1}$ National Institute of Science, Technology and Development Studies (NISTADS), New Delhi, India Correspondence: (email: pgoswami@nistads.res.in)
} 


\section{M}

Introduction any social systems can be generically represented as a collection of individuals with varying degrees of performance, with diverse responses to different drivers. While quantitative descriptions of the evolution of such systems can be helpful in efficient goal-oriented management, mathematical modelling of such systems presents challenging problems. One of the major challenges is that unlike in physical systems, it is difficult to identify unambiguous, let alone unique, casual relations in social dynamics. Another major difficulty is in quantifying attributes like performance, personal choice and leadership. In particular, a dynamical (mathematical) model requires precise quantitative descriptions of aspects that may be often subjective. While the importance of social dynamics has been recognized for some time (Allport, 1924; Newcomb et al., 1965; Tuma and Hannan, 1984) and continues to be emphasized (Matthies et al., 2001; Mogilner et al., 2003; Bejan and Merkx, 2007; Castellano et al., 2009), there have not been many attempts at quantitative dynamical descriptions. A primary reason, in addition to the challenges discussed above, is likely attributable to the slow realization of the potential of mathematical modelling of such systems aided by powerful computing. One area that has received strong attention for some time is institutional economics, on which a large number of studies have focused (Bardhan, 1989; Hodgson, 2000; Rutherford, 2001; Chavance, 2008), as well as various aspects related to the effects and management of funding (Commons, 1934; Hodgson, 1998; Schotter, 2008), in addition to its current structure (Rutherford, 2001). There have been also attempts to relate institutional economics to social processes (Granovetter, 1985; Hodgson, 1993; White et al., 2004), and description of institutional processes, such as inter-personal relations (Heider, 1982), role of culture (Maanen and Barley, 1984; Harrison and Carrol, 2002) and response (Cronbach, 1955), but mostly in qualitative frameworks. The potential of applying mathematical concepts and modelling to biological systems has also been recognized (Gueron et al., 1996; Brauer and CastilloChavez, 2001; Edelstein-Keshet, 2004); in addition, there is growing interest in modelling connected biological and social networks (McPherson et al., 1992; Hegselmann and Flache, 1998; Skyrms and Pemantle, 2000; Barabasi et al., 2002; Antal et al., 2005; Facchetti et al., 2011; Chen et al., 2013; Baer et al., 2015). Similarly, there have been attempts at quantitative and conceptual modelling of certain social processes (Thalheim, 2012) like group dynamics (Gueron et al., 1996; Flierl et al., 1999; Palla et al., 2007; Vugt and Schaller, 2008) and specific events like elections
(Fieldhouse et al., 2007; Nagel, 2010) and acts of terrorism (Allanach et al., 2004; Farley, 2007). In general, the potential application of mathematical modelling in assessing the performance and dynamics of organizations has been explored less mostly because of the difficulties indicated.

Many industrial or business organizations are characterized by short-term specific goals in terms of production, sales and profit; in particular, the goals and the targets of such organizations can change quickly and abruptly. Research institutes represent a special class of organization in that they have long-term goals and somewhat loosely defined targets and thus are primarily driven by individual performers within the broad institutional objectives (Carrol and Burton, 2007). A research organization can be also viewed as an interactive social network, with attitudes and responses subject to a spectrum of factors. A characteristic feature of many structured organizational systems is an interaction with the institutional (social) environment; the social environment itself can change due to collective inputs from its members (McPherson et al., 1992; Chen et al., 2013). A quantitative description of some of these processes is challenging, but attemptable. It has been shown that the distribution of members' attitudes to a controversy can be given a quantitative description (Abelson, 1964); however, unlike many social organizations, the structure and interaction of a research organization require special attention (Cronbach, 1955; Heider, 1982; Maanen and Barley, 1984; Skyrms and Pemantle, 2000).

A general characteristic of a research organization (unlike, for example, an electorate), if not always in practice, is that, by definition, it is governed by a top-down approach with a leadership that identifies the broad objectives and resource availability. Of course, this top-down input is generally at a policy level and not at the level of implementation. However, identification of research priorities involves a top-down process, with an effective and interactive feedback from the bottom-up. In terms of publication (performance), research organizations across the world are generally free to publish their results from (at least) non-strategic research; however, a top-down process is involved in aspects like resource allocation. Even the top-down input may not be exactly an explicit and direct control; however, identification of priority programmes, areas of particular national interest and peer pressure automatically bring in certain topdown inputs. While a scientist may define research goals and apply for funds, the availability of such funds will depend on the policy and perception of the funding agencies like Government and industry. This top-down control is subtle but powerful.

Table 1 | Description and range of parameters

\begin{tabular}{|c|c|c|c|}
\hline \multirow[t]{2}{*}{ Parameter } & \multirow[t]{2}{*}{ Symbol } & \multicolumn{2}{|c|}{ Values } \\
\hline & & Standard & Range \\
\hline Coefficient of restoration to maximum & $\alpha$ & 0.00002 & $0.00001-0.00004$ \\
\hline Coefficient of restoration to average & $\beta$ & 0.000001 & $0.0000000001-0.001$ \\
\hline Coefficient of restoration to national benchmark & $\delta$ & 0.0000002 & $0.000000001-0.001$ \\
\hline Strength of interaction with leadership & $C_{R}$ & 0.2 & $0.1-0.4$ \\
\hline Initial selection criteria & $C_{1}$ & 1.0 & $0.5-2.0$ \\
\hline National benchmark & $X_{N}(0)$ & 2.0 & - \\
\hline Global benchmark & $X_{G}$ & 2.0 & - \\
\hline \multicolumn{4}{|l|}{ Threshold for selection: } \\
\hline Low threshold & $T_{L}$ & $0.1^{\star} X_{G}$ & - \\
\hline High threshold & $T_{H}$ & $0.8^{\star} X_{G}$ & - \\
\hline Adopted benchmark & $x_{0}$ & 2.0 & - \\
\hline
\end{tabular}


A challenging task is to represent and integrate such top-down (external) inputs into the institutional dynamics.

A research organization may be also viewed as a large-scale (signed) connected network (Iacono and Altafini, 2010) and treated accordingly; however, we believe a dynamical description, with appropriate representations of forcings, is more appropriate due to its similarity with a time-dependent evolving system. The dynamical description will have to also account for group dynamics such as the effect of the group size (Bond, 2005; Toral and Tessone, 2007), attitude and response under competing factors (Cronbach, 1955), role of the restoring influences (Friedman and Friedman, 1984; Galam, 2004) and others. In particular, we consider a research organization as a dynamical system with initial conditions determined by initial selection criteria, with evolution governed by various "forces".

A reasonable and quantitative measure of performance of a scientist engaged in non-strategic research (that is, where research results are not subject to restrictions for publications) is the number of publications in journals with Science Citation Index (SCI). To avoid ambiguity without making the criteria too restrictive, we shall interpret the dynamical variable as the number of publication in any SCI journal to be of equal value; inclusion of other metrics like impact factor of journal and citation of publications is straightforward and not considered at this stage.

The primary objective of this work is thus to formulate a mathematical description of the dynamics of a research organization, with the performance of an individual as a dynamical

\begin{tabular}{|lccc|}
\hline \multicolumn{4}{|l|}{ Table 2 | Three sets of representative scenarios } \\
Parameter & Set 1 (S1) & Set 2 (S2) & Set 3 (S3) \\
\hline$\alpha$ & 0.0002 & 0.0002 & 0.00002 \\
$C_{R}$ & 0.5 & 0.2 & 0.2 \\
$C_{I}$ & 1.4 & 1.0 & 1.0 \\
$\gamma$ & 0.01 & 0.01 & 0.001 \\
\hline
\end{tabular}
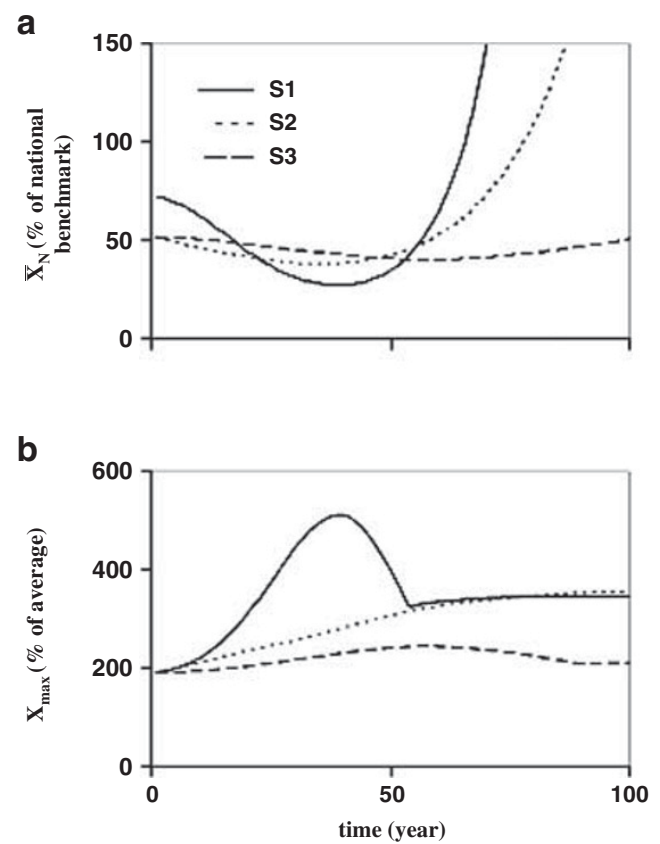

variable; the overall institutional performance is thus built up in a bottom-up manner. It should be emphasized that the model is concept-based, rather than data-based.

\section{Methodology}

The dynamical institutional model. To a large extent, a research and development organization acts like a constrained dynamical system with internal feedbacks and initial value; the more general case is that of an initial-boundary value system. The initial value is determined by the selection criteria set in the beginning generally in terms of some national or global benchmarks. While there would be subsequent inductions, the performance of the first batch as well as the selection criteria provide benchmarks and restoring forces that determine the evolution of the system. Behaviour of the social groups also depends on the size of the group (Bond, 2005; Toral and Tessone, 2007). Because we are considering averages over individual performances, smaller or larger numbers may affect the results. However, for a research organization, this size is expected to be within some logical limits; thus we need to only consider groups of small sizes, typically between 50 and 200. We do not consider the case of firing of an employee due to low performance; such a situation is generally rare for research organization. The leadership response is primarily in terms of strength of interaction (attention). However, employees may leave and new recruitment will take place. We thus consider, for simplicity, the total number to remain constant in time.

Drivers and restoring forces. Each individual is expected to be affected by various social processes like the nature of the work (Maanen and Barley, 1984), differences in opinions (Maanen and Barley, 1984) and inter-personal relations (Heider, 1982). However, it is also expected that these responses will be somewhat systematic (Abelson, 1964) and quantitatively representable. As discussed above, the dynamical variable considered is the individual performance in terms of the number of SCI

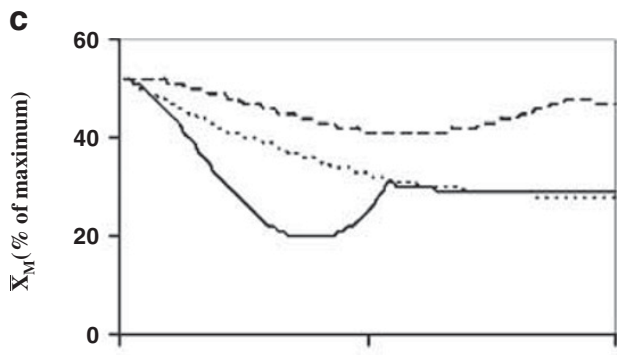

d

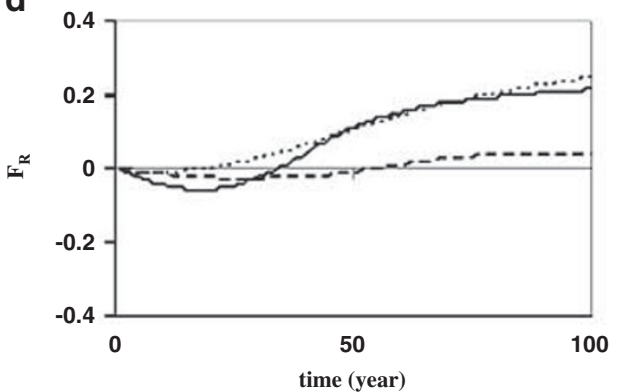

Figure 1 | Evolution of the performance parameters under different institutional environments in terms of average institutional performance as percentage of national benchmark (a), maximum performance as percentage of institutional average performance (b), institutional average performance as percentage of maximum performance (c) and response of leadership (d) represented by the three sets of parameters (S1, S2, S3). The dynamics represented by the sets S1 and S2 are unrealistic while that represented by S3 (adopted as standard set) is stable. 
publications per year; this individual performance is assumed to be affected by

(a) The difference between the individual performance and the highest performance and is assumed to act both as a restoring and a driving force in the form of a motive force, represented by

$\alpha\left[X_{\max }(t)-x_{i}(t)\right]$

Here $X_{\max }(t)$ represents the highest performance of the institute while $x_{i}(t)$ represents the individual performance at the time $t$. While this force drives the performer to reach $X_{\max }(t)$, closeness to $X_{\max }(t)$ induces complacency; at large difference from $X_{\max }(t)$ (small $x_{i}(t)$ ), the forcing is large. The coefficient $\alpha$ determines the strength of this effect and will implicitly depend on institutional policies for evaluation of performance.

(b) The average performance of the institute: The difference between the individual and the average performance acts like a restoring factor, with a "spring" constant characteristic of the institute. Thus departure from the institutional average performance creates a restorative force, also because of the tendency on the part of many to resist change (Friedman and Friedman, 1984; Galam, 2004). This factor is represented as

$\beta\left[x_{i}(t)-\bar{X}(t)\right]$

where $\beta$ is a coefficient that determines the effect of this factor, and

$x_{i}(t)$ : Performance of the member $i$ at time $t$.

$\bar{X}(t)$ : Average performance of the group at time $t$.

(c) Departure from National/Global standards: It is assumed that performance above national (global) benchmark generally slows down the initiative for higher performance due to complacency; thus the effect of this is represented as

$$
\begin{aligned}
& \gamma\left[x_{i}(t)-X_{G}\right] \\
& \delta\left[x_{i}(t)-X_{N}\right]
\end{aligned}
$$

This forcing can be logically expected to be dynamic due to global information network. The coefficient $\gamma$ determines the strength of departure from individual performance to global standards while the coefficient $\delta$ determines the strength of departure from individual performance to national standards. The term $X_{N}$ and $X_{G}$, respectively, represent benchmark of performance at national and global level. Typically, they may be represented as national and international average of SCI publications. Thus, in principle, both $X_{N}$ and $X_{G}$ are themselves dynamical variables; however, for simplicity we consider fixed and representative values of these parameters (Table 1). Extension to include variable benchmarks is straightforward.

Thresholds of performance and categories of performers. We define two thresholds as percentage of global benchmarks, to define cut-offs:

$T_{L}=\varepsilon_{L} * X_{G}$

$T_{H}=\varepsilon_{H} * X_{G}$

Here $T_{L}$ and $T_{H}$, respectively, represent the thresholds of lower performance and higher performance. The coefficient $\varepsilon_{L}$ and $\varepsilon_{H}$, respectively, represent the strength of threshold. The value of global benchmark is given in Table 1 .

We then define three categories of performers in terms of these cut-offs:

Non-performers $\left(N_{N}\right): x_{i}(t) \leqslant T_{L}$

Intermediate performers $\left(N_{I}\right): T_{L} \leqslant x_{i}(t) \leqslant T_{H}$

High performers $\left(N_{H}\right): x_{i}(t)>T_{H}$
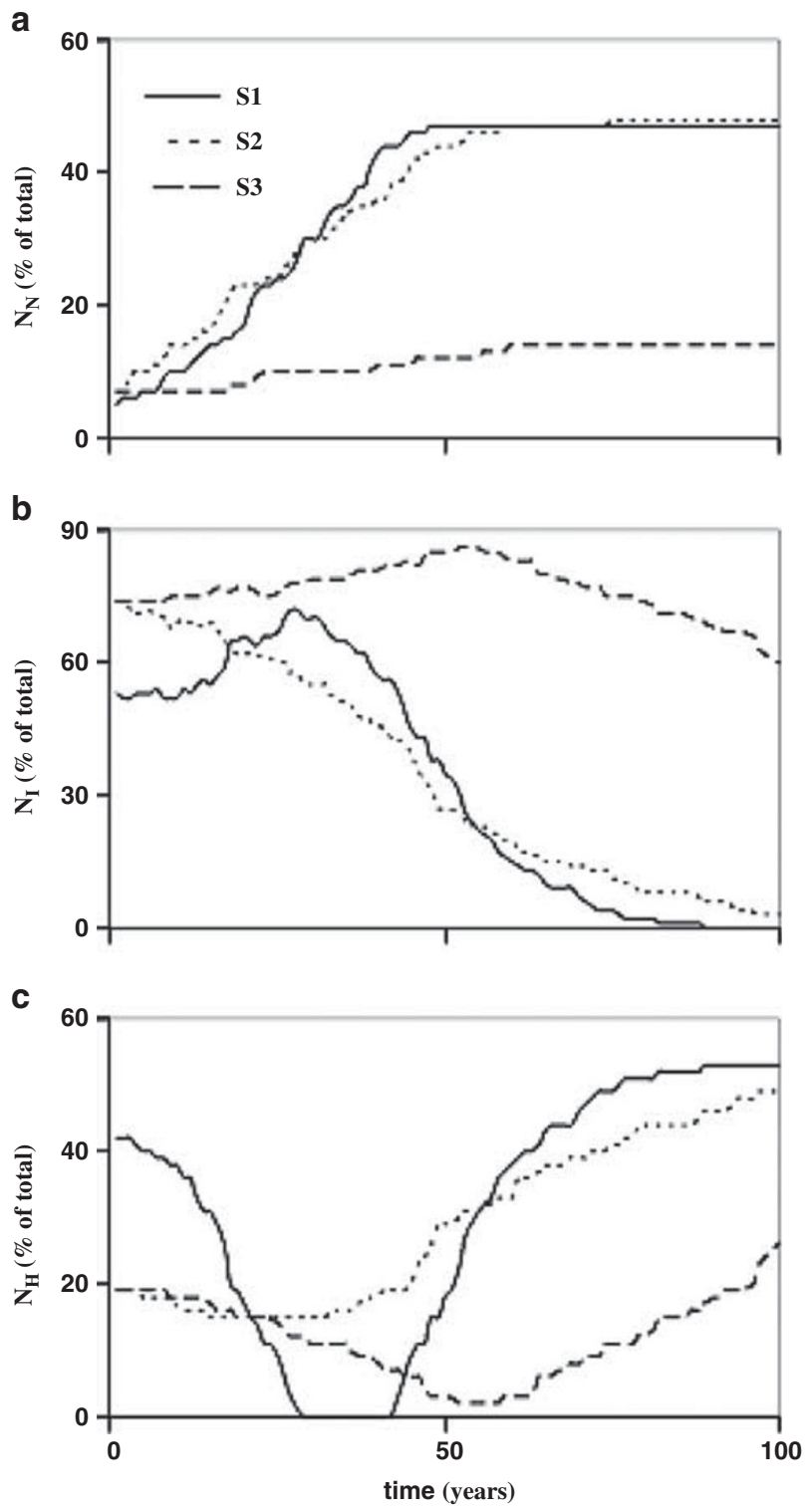

Figure 2 | Evolution of non-performers $\left(N_{N}\right)(a)$, intermediate performers $\left(N_{l}\right)$ (b) and high performers $\left(N_{H}\right)(c)$ as a percentage of the total number (100) in the group for different institutional environment defined by the three sets of parameters (S1, S2, S3). For institutional environment characterized by the sets $S 1$ and S2, the evolution of performers in different categories exhibit unrealistic behaviour; for set S3, the evolution and the distribution of performers in different categories are realistic.

The coefficients used to define the categories are adopted as $\varepsilon_{L}=0.5$ and $\varepsilon_{H}=1.5$.

The dynamical model and initial condition. Combining these factors we write the equation for the dynamics of individual performance as

$$
\begin{aligned}
\frac{d x_{i}(t)}{d t}= & \alpha\left(X_{\max }(t)-x_{i}(t)\right)+\beta|x(i)-\bar{X}(t)|+\gamma\left(x_{i}(t)-X_{G}\right) \\
& +\delta\left(x_{i}(t)-X_{N}\right)+C_{R} * R(t) * x_{i}(t)
\end{aligned}
$$

where $x_{i}(t=0)=x_{i}(0)$.

Here the last terms represent the response of the leadership to the $i$ th performer, and $C_{R}$ defines the strength of the interaction 
a

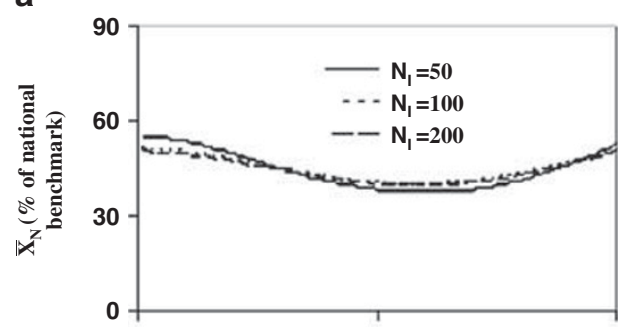

b

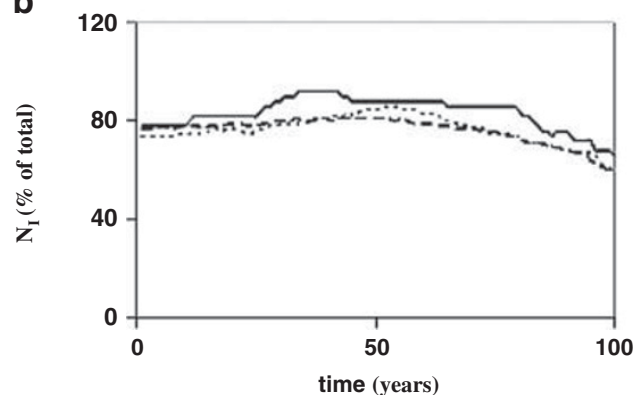

C

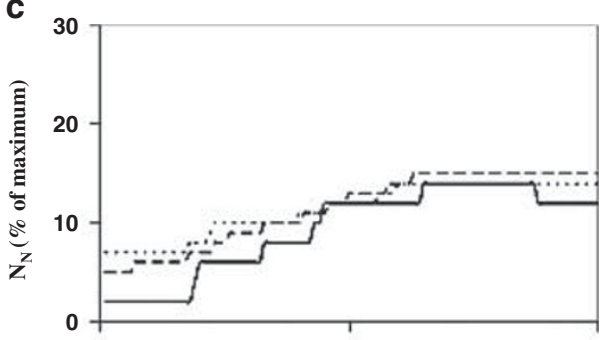

d

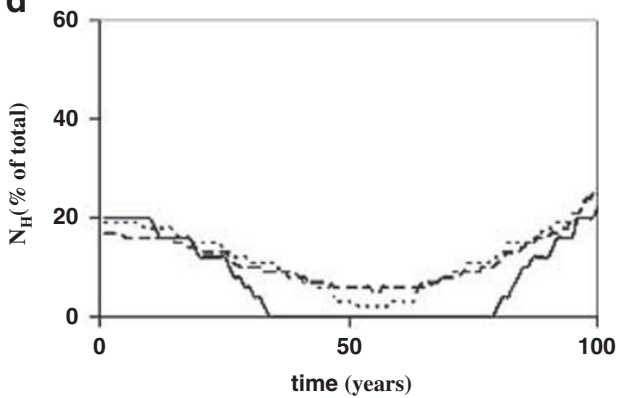

Figure 3 | Impact of the size (number of members; $N$ ) of the group on the dynamics of average performance as percentage of national benchmark (a) and the categories of performers: non-performers $\left(N_{N}, b\right)$, intermediate performers $\left(N_{l}, \mathrm{C}\right)$ and high performers $\left(N_{H}, d\right)$ as percentage of the total number of an institution for the standard set of parameters that define the institutional environment. The dynamics is not very sensitive to the size of the group; however, for a size of $\geqslant 200$, the behaviour becomes somewhat unrealistic.

between individual member and the leadership, characterized by the response function, $R(t)$; it is assumed to be proportional to the performance of the $i$ th member.

For consistency, we impose the condition $x_{i}(t) \geqslant 0$ in the simulations, and apply equations (1)-(4), accordingly.

The performance of an individual is also influenced by the nature and strength of the interaction with the leadership (approving authority). This response $R(t)$ of the leadership is considered a dynamical variable in response to the strength of interaction with the performers in two categories:

$\frac{d R}{d t}=C_{R H} N_{H}-C_{R L} N_{N}$

Here $R(t)$ represents the overall response of the leadership based on approach and policy towards high and low performers, characterized by the coefficients $C_{R H}$ and $C_{R L}$, respectively.

It is assumed that the initial intake is based on certain quantitative quality criteria such as the number of years of experience, number of SCI publications and teaching. In our case, we express this criterion in terms of the number of SCI publications at the initial time of induction:

$x_{i}(t=0) \geqslant T_{S} * X_{N}(0), \quad T_{S} \leqslant 1$

where $T_{S}$ defines a threshold and $X_{N}(0)$ represents national average in $x$ at time $t=0$. This representation assumes that recruitment of scientistsis at different levels, with a minimum entry qualification $\left(T_{S}{ }^{\star} X_{N}(0)\right)$. We represent initial conditions as

$$
\begin{aligned}
& x_{i}(t=0)=r(i) * C_{I} * X_{0} \\
& x_{i}(t=0) \geqslant X_{0}
\end{aligned}
$$

where $X_{0}$ is an adopted benchmark and $C_{I}$ is the coefficient of selection and $r(i)$ is a random number that varies with the individual. The values of $C_{I}$ are considered as greater and equal to 1 for three scenarios (Table 2). The values of $X_{N}(0)$ and Ts are given in Table 1. The random number is introduced to incorporate unavoidable variability in application of selection criteria; the range of $r(i)$ was limited to between 0.8 and 1.0 to ensure minimum quality. The ranges of $C_{I}$ and $X_{0}$ are chosen to ensure overall consistency of equations (9) and (10).

The primary dynamical variable, namely, the number of SCI publications by an individual in a year, is naturally an integer. However, without any loss of generality, we have treated it as a general real number. This allows us to treat various other (fractional) quantities like the averages in the equation on a single footing. Besides, it also allows, at a later time, to allow fractional publication based on criteria like the number of authors involved in a publication.

Analysis parameters. While many performance indices are relevant and can be defined, we have considered a single variable, interpreted as the number of SCI publications of an individual, for our analysis.

The average institutional performance is defined by

$\bar{X}(t)=\frac{1}{N} \sum_{i=1}^{N} x_{i}(t)$

Here $N$ represents the total number of individuals involved in assessing the performance of an RDI. The maximum performance of an RDI at any given time is defined as the maximum of $\left\{x_{i}(t), i=1, \ldots \ldots, N.\right\}$. The average institutional performance as percentage of global/national benchmark and the maximum performance are calculated as

$$
\begin{aligned}
\bar{X}_{G}(t) & =\frac{\bar{X}(t)}{X_{G}} * 100, \quad \bar{X}_{N}(t)=\frac{\bar{X}(t)}{X_{N}} * 100, \quad \bar{X}_{M}(t) \\
& =\frac{\bar{X}(t)}{X_{\max }} * 100
\end{aligned}
$$

Here $\bar{X}_{G}(t)$ and $\bar{X}_{N}(t)$, respectively, represent the average institutional performance as percentage of global benchmark and national benchmark while $\bar{X}_{M}(t)$ represents the average institutional performance as percentage of maximum performance. 

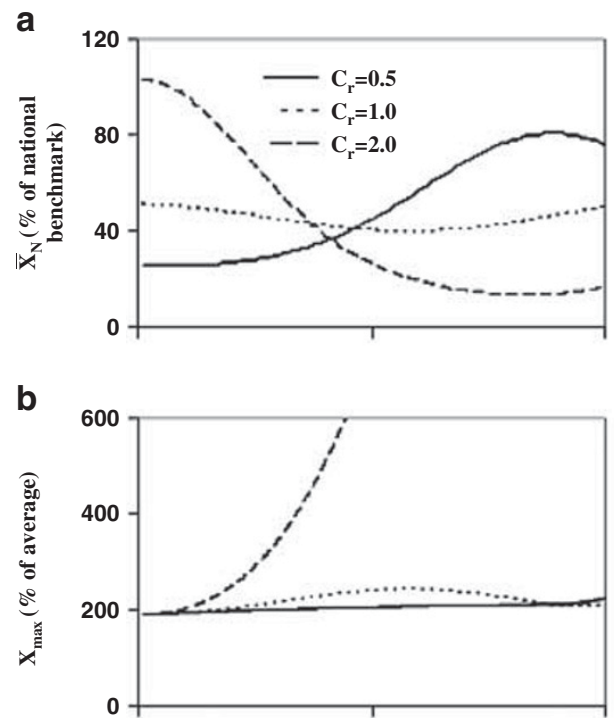

C

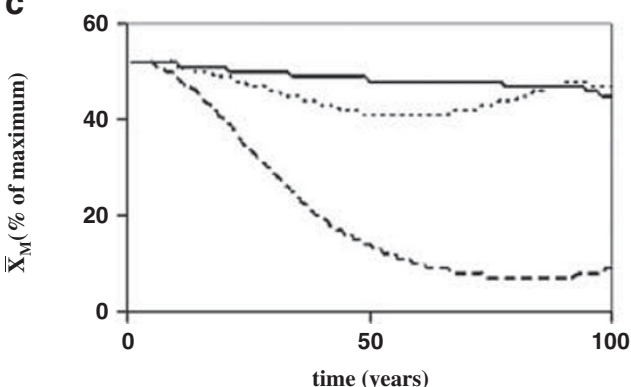

d

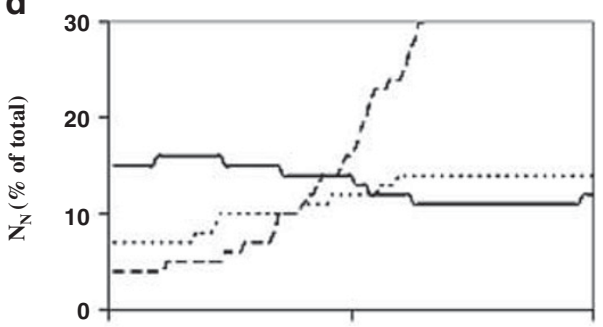

e

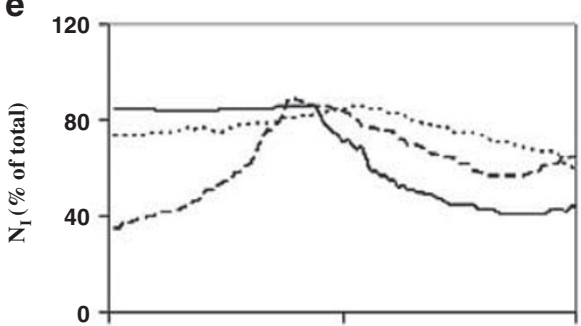

$\mathbf{f}$

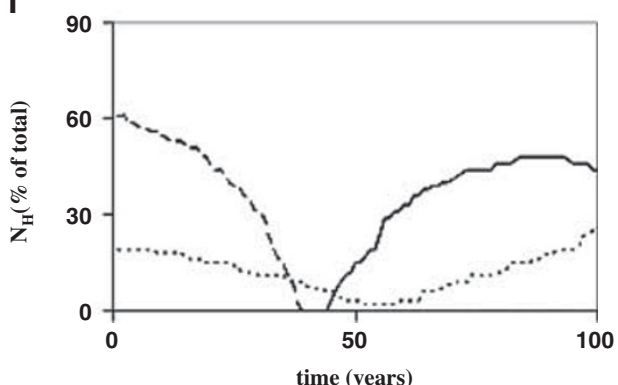

Figure 4 | Impact of initial selection criteria (cut-off in terms of national benchmark) on institutional dynamics for three values of cut-offs. Left panels: average performance as percentage of national bench mark (a), maximum performance as percentage of institutional average performance (b) and institutional average performance as percentage of maximum performance (c).

Right panels: Categories of performers: non-performers $\left(N_{N}\right)(d)$, intermediate performers $\left(N_{l}\right)(e)$ and high performers $\left(N_{H}\right)(f)$ as percentage of the total number.

As expected of a complex dynamical system, the evolution shows strong sensitivity to the initial conditions.

The percentage of performers in three categories is calculated as per equation (6). For convenience, we shall express the number of high performers, intermediate performers and non-performers as percentage of the total number based on equation (6).

Calibration and reference state. In the absence of constitutive relations or theoretical or observational constraints, the set of parameters describing the system (Table 1) cannot be ascribed unique or precise values. Similarly, performance data on individuals over long periods are not easily available or accessible for validation. Instead, our approach is to consider a set of values that produce "reasonable" behaviour and examine the system behaviour against this reference state due to various processes. Thus, although the parameters can be in principle arbitrary, they are constrained to a range around $0.5-2.0$ (Table 1) around their calibrated values to study their impact. The range adopted for a parameter also depends on how sensitive the results are to the parameter.

The behaviour of the system is then studied under different initial distributions as well as varying strengths of the coefficients. To examine the sensitivity of the system to different combinations of the restoring forces, we have considered three sets S1, S2 and S3 (Table 2). On the basis of the general system behaviour, discussed below, a set of parameters was adopted as the reference state for analysis.

\section{Results}

Here we first present certain broad features on the dynamics of the system, followed by an analysis of relative roles of various processes.

Evolution of general system behaviour and the reference state. The average performance, as a percentage of national benchmark $\left(X_{N}\right)$, shows (Fig. 1, top left panel) complex but distinct behaviour based on the combination and the strengths of the coefficients in terms of the three sets S1, S2 and S3 (Table 2). Thus for set S1, the average performance shows a decline from approximately $60 \%$ of the national benchmark for the first 50 years, with a minimum around 50th year; after approximately 50 years an exponential growth begins (Fig. 1, top left panel). For the set S2, the average performance remains close to $100 \%$ of the national average for about 60 years, after which it shows exponential growth (Fig. 1, top left panel). For the parameter set S3, the institutional performance remains close to the national average essentially for all time up to 100 years (Fig. 1, top left panel). Similarly, the average performance, as a percentage of institutional maximum, shows (Fig. 1, top right panel) complex evolution in terms of the three sets S1, S2 and S3. For set S1, $\bar{X}_{M}$ decreases up to about 50 years, after which it remains essentially stable around $30 \%$; for both S2 and S3, the average performance in terms of institutional maximum is essentially stable beyond approximately 50 years (Fig. 1, top right panel). However, for the set S3, the values of $\bar{X}_{M}$ 
a

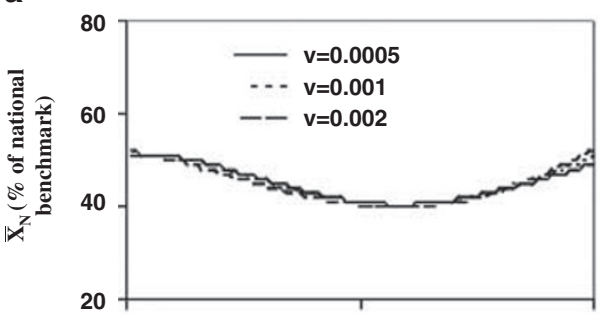

b

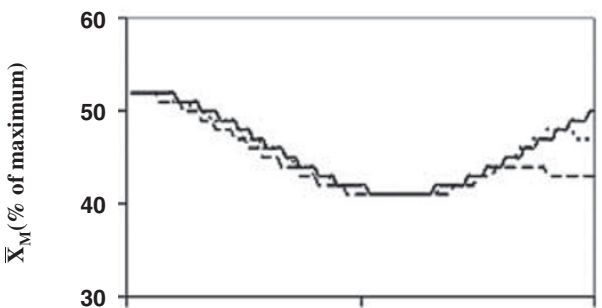

C

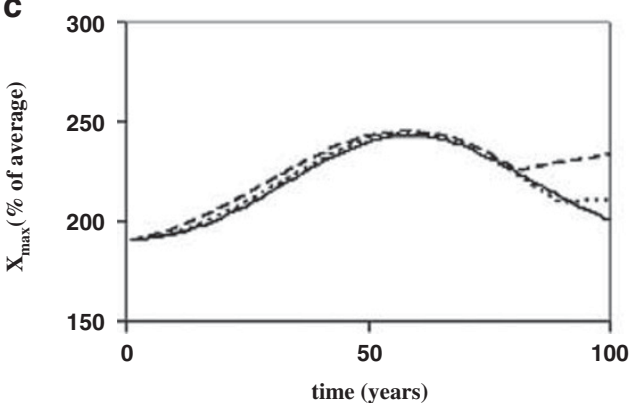

d

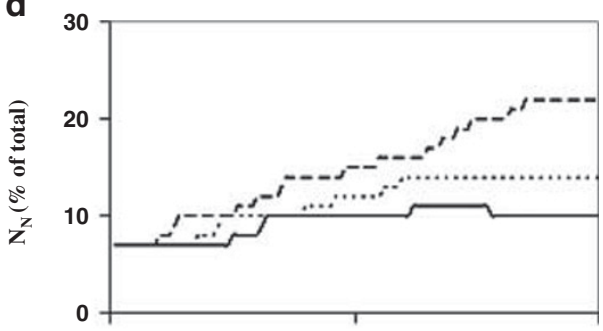

e

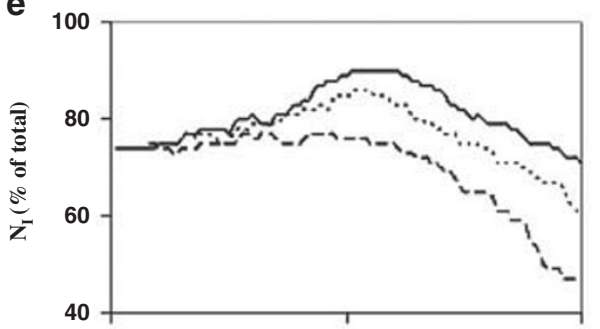

$\mathbf{f}$

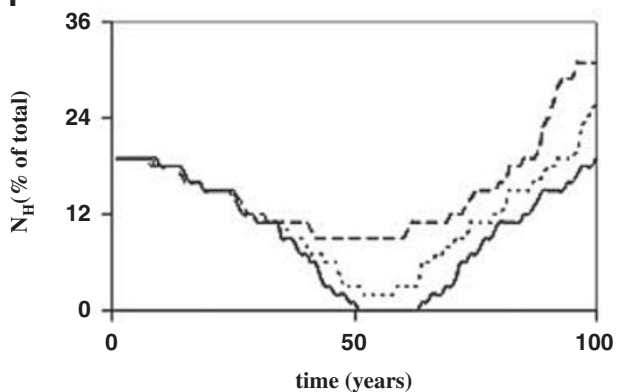

Figure 5 | Impact of strength of coefficient of global benchmark on the institutional dynamics in terms of average performance as percentage of national benchmark (a), maximum performance as percentage of institutional average performance (b) and institutional average performance as percentage of maximum performance (c) (left panels) and the number of non-performers $\left(N_{N}\right)(d)$, intermediate $\left(N_{l}\right)(e)$ and high performances $\left(N_{H}\right)$ (f) respectively as percentage of the total number (right panels). The institutional dynamics in terms of average and maximum are not sensitive to the global benchmark; however, evolution of performers in different categories shows strong response to global benchmark.

are generally above $40 \%$. The maximum performance $\left(X_{\max }\right)$, as percentage of institutional average, also shows complex but different evolution paths depending on the combination and the strengths of the coefficients (Fig. 1, bottom left panel). For set S1, this increases up to about 50 years and then stabilizes. For both sets S2 and S3, the maximum performance remains nearly constant up to 100 years. Similarly, the magnitude of response of leadership declines mildly and then stabilizes for both S1 and S2 (Fig. 1, bottom right panel); in contrast, the leadership response function for set S3 has similar magnitude throughout 100 years.

The growth of the three categories of performers high $\left(N_{H}\right)$, intermediate $\left(N_{I}\right)$ and low $\left(N_{L}\right)$ for the three sets can be very different and striking; in particular, the system can generate very high to very low number of non-performers (Fig. 2) depending upon the strengths of the parameters. For S1, the number of nonperformers increase up to about 50 years, followed by a nearly constant value around $40 \%$; for S2, the behaviour is very similar. In contrast, this number is nearly constant and does not exceed $20 \%$ for S3 (Fig. 2, top panel). For the sets S1 and S2, the number of high performers is generally higher in the later years (Fig. 2, bottom panel), with a corresponding decline in the number of intermediate performers (Fig. 2, middle panel); for set S3, on the other hand, the fraction of high performers declines in the beginning but increases after the 50th year (Fig. 2, bottom panel); all throughout the 100 years, the set S3 produces 60-90\% intermediate performers. In what follows, we shall consider the parameters for set S3 as our standard set of parameters (Fig. 2).

As discussed, the total number of scientists in a research organization is not expected to vary widely. It was also verified that the system behaviour in terms of parameters considered did not depend on the size of the group in any appreciable manner (Fig. 3); however, this conclusion does not necessarily apply to other aspects of group dynamics. In what follows, therefore we shall consider the set S3 for a group of 100 to investigate response of the system to various processes considered (Fig. 3).

We next examine the three parameters of system performance: institutional average as percentage of national benchmark $\bar{X}_{N}$, institutional maximum as percentage of institutional average $X_{\max }$ and average performance as percentage of maximum $\bar{X}_{M}$ along with the three categories of performers.

Impact of initial selection criteria. The initial selection criteria $\left(C_{I}\right)$ have strong effect on the average performance, and especially on the maximum performance (Fig. 4). Except for large values of $\mathrm{C}_{\mathrm{I}}$, the evolution is bounded (Fig. 4, top left panel); however, for maximum performance as percentage of institutional average (Fig. 4, left middle panel), the impact of initial selection is 
a

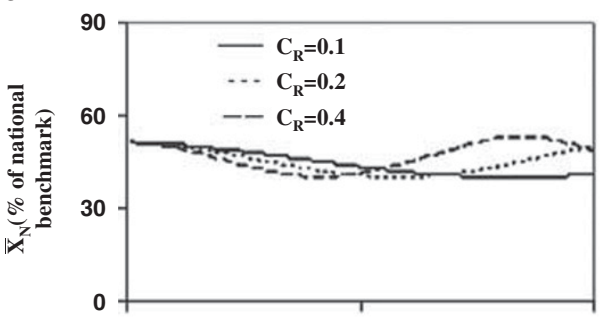

b

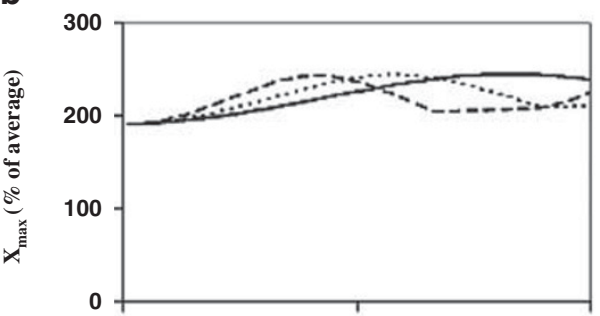

C

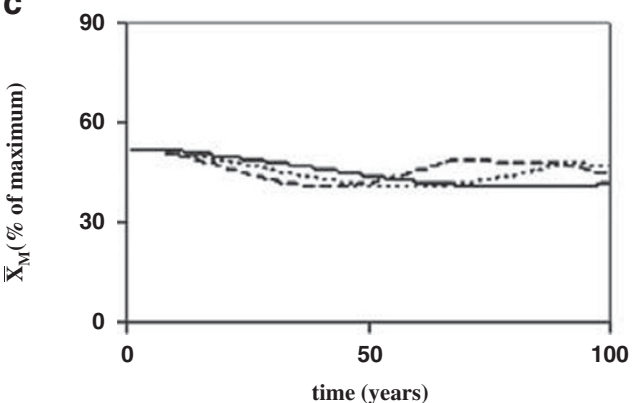

d

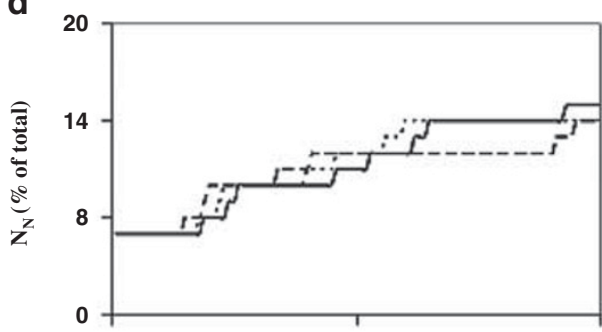

e

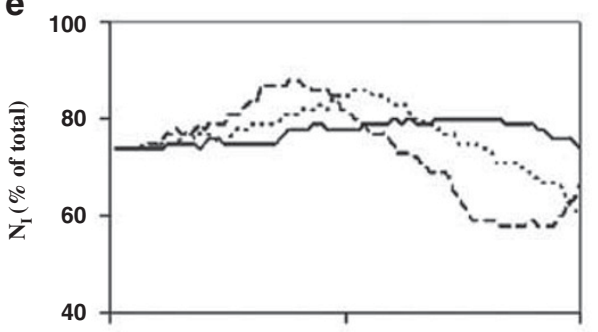

f

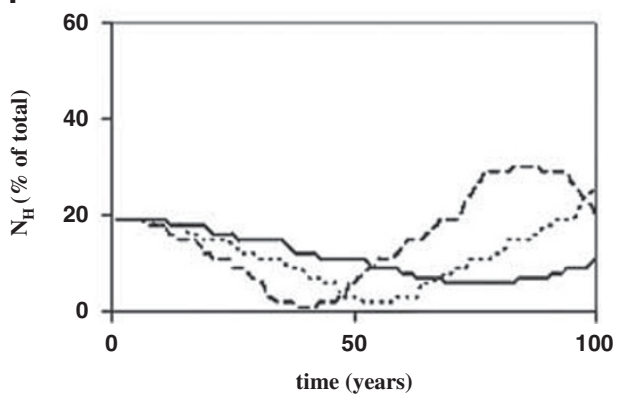

Figure 6 | Impact of the strength of the response $\left(C_{R}\right)$ of the leadership on the members in the evolution of institutional dynamics in terms of average performance as percentage of national benchmark (a), maximum performance as percentage of average performance (b) and average institutional performance as percentage of maximum performance (c) (left panels) and the numbers of non- $\left(N_{N}\right)(d)$, intermediate $\left(N_{I}\right)$ (e) and high performers $\left(N_{H}\right)(f)$ as a percentage of the total number (right panels). The institutional performance in terms of average and maximum does not show much sensitivity to strength of response of leadership; however, response of leadership strongly affects the number of performers, especially the number of high performers.

dramatic for $C_{I}=2.0$. Consistently, the institutional average as percentage of maximum performance shows similar but reverse behaviour during these years. Similarly, the initial selection criteria strongly affect the distribution of the participants in different performance categories (Fig. 4, right panels) even at later years. Thus for smaller values of $C_{I}$, both the number of non-performers (Fig. 4, top right panel) and the number of high performers are small up to about 50 years; the system is dominated ( $>80 \%$ ) by intermediate performers (Fig. 4, middle right panel); however, the number of high performers rises beyond 50 years to saturate around $50 \%$ (Fig. 4, bottom right panel). For high selection criteria, conversely, the number of high performers begins with large values but declines; at the same time, the number of nonperformers begins with a small $(<10)$ percentage but rises sharply (Fig. 4).

Relative roles of a processes on institutional performance. Since our system is characterized by a number of parameters, it is necessary to examine sensitivity of the results to these parameters. It was found that the results, in terms of average institutional performance as percentage of national benchmark $(\delta)$, percentage of institutional maximum, were not very sensitive to the thresholds $\left(T_{L}\right.$ and $\left.T_{H}\right)$. Also, for a wide range of the parameters, the institutional dynamics produced similar behaviour
(Supplementary Figs 1-4). Similarly, the results did not vary in any appreciable manner (not shown) with changes in the strengths of restoration to institutional maximum $(\alpha)$, or institutional average $(\beta)$. The results were also verified to be not sensitive to the values of low and high cut-off $\left(\varepsilon_{L}\right.$ and $\left.\varepsilon_{H}\right)$ used to define the response function (Supplementary Fig. 5 and 6).

Role of restoration to benchmark. Because the thresholds for performance are defined in terms of global benchmark $\left(X_{G}\right)$, the strength of contribution of global benchmark $(\gamma)$ is expected to have strong effects on institutional dynamics. In particular, the strength of global benchmark determines the number of nonperformers and intermediate performers; this, in turn through the leadership response (equation 4) can affect the institutional dynamics. Indeed, the growth in the percentage of the three categories of performers was found to vary with the strength of $\gamma$, especially in the latter years.

Variations in the strength of restoration to global benchmark, characterized by $\gamma$, do not have any appreciable effects on the average performances $\bar{X}_{N}, \bar{X}_{M}$ and $X_{\max }$ (Fig. 5, left panels). However, the categories of performers can change appreciably, especially after about 50 years, with the strength of restoration to global benchmark (Fig. 5); interestingly, both weaker and stronger 
a

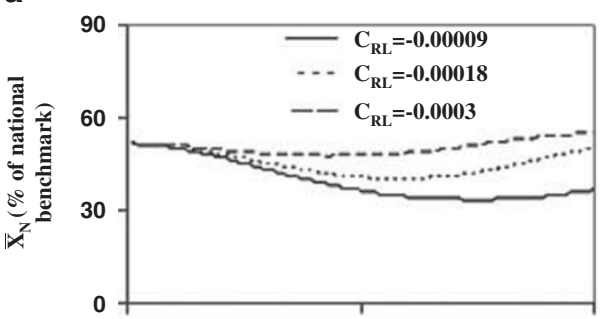

b

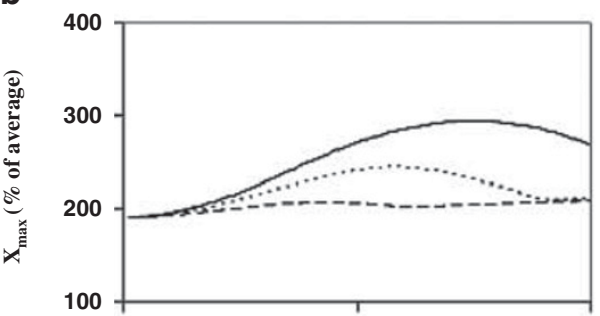

C

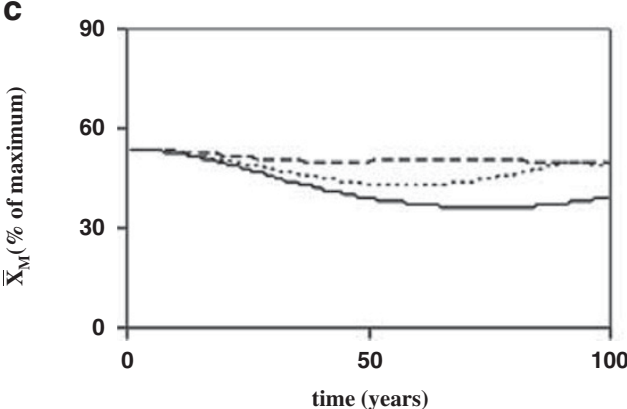

d

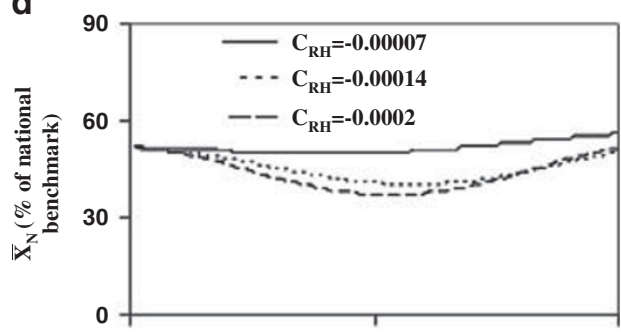

e

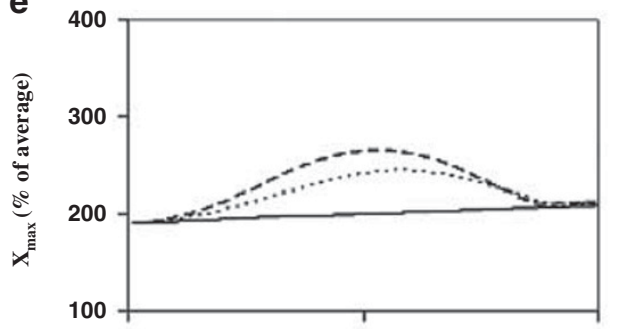

$\mathbf{f}$

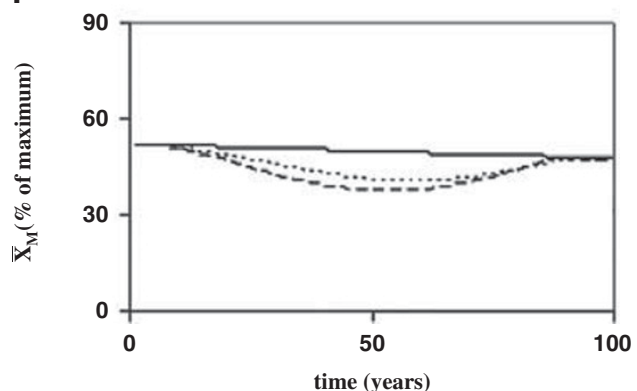

Figure 7 | Impact of differential response of leadership to low and high performers on institutional dynamics in terms of average and maximum performance. Left panels: Impact due to variations in strength of response to low performers $\left(C_{R L}\right)$. Right panels: Impact due to variations in strength of response to high performers $\left(C_{R H}\right)$. The differential response of leadership has less effect on the institutional average and the institutional maximum in the initial years but strong in the later years.

strengths of $\gamma$ result in lower number of non-performers and high performers (Fig. 5, right panels).

Strength of interaction with leadership. The parameters like $\bar{X}$ and $X_{\max }$ do not show significant sensitivity to the strength of leadership response (Fig. 6, left panels). Similarly, the number of non-performers is not significantly affected by the strength of response of leadership. However, it has significant effects on the numbers of intermediate and high performers (Fig. 6, right panels). In particular, stronger interaction with leadership enhances the number of high performers, especially in the later years (Fig. 6, bottom right panel).

The differential response to low and high performers turns out to be a critical factor in determining institutional performance. Variations in the strength of response to low performers can result in different values and evolution of institutional performance (Fig. 7, left panels). Interestingly, the changes in the institutional performance due to variations in the strength of response to high performers generally produce effects opposite to those due to variations in the strength of response to low performers (Fig. 7, right panels). For high response to non-performers (characterized by $C_{R L}$ ), the percentage of non-performers grows to approximately $50 \%$ and saturates (Fig. 8, left top panel). Interestingly, the corresponding intermediate performers (Fig. 8, left middle panel) decrease, while the percentage of high performers remains nearly steady around $50 \%$ (Fig. 8, left bottom panel). Weaker response to non-performers can bring down the percentage of non-performers to small values, with a corresponding gain in the percentage of high performers (Fig. 8, left panels). Stronger response to high performers (characterized by $C_{R H}$ ) expectedly results in a reduction of non-performers (Fig. 8, top right panel). However, the most dramatic effect of variation of strength in response to high performers is seen in the percentage of high performers; for a value of $C_{R H}=-0.00007$, the percentage of high performers grows to nearly $90 \%$ and stays steady for a long time (Fig. 8 , right bottom panel).

The response of the leadership $\left(F_{R}\right)$, both total (characterized by $C_{R}$ ) as well as differential (characterized by $C_{R H}$ and $C_{R L}$ ) show complex and interesting variability in time; in particular, $F_{R}$ shows a certain periodicity for a wide spectrum of values of $C_{R}$, $C_{R H}$ and $C_{R L}$ (Fig. 9). While the periodicity of $F_{R}$ depends on these parameters, the phenomenon is quite robust, indicating presence of intrinsic regimes.

\section{Discussion}

Given that the outcome of R\&D programmes may only be realized after several years of sustained and coordinated, research effort and associated high investment, the long-term performance of research organizations is particularly important. At the same time, such R\&D output can have critical and transformative impact on wider society, and as such it is necessary to identify time scales of evolution and unavoidable loss in performance 
a

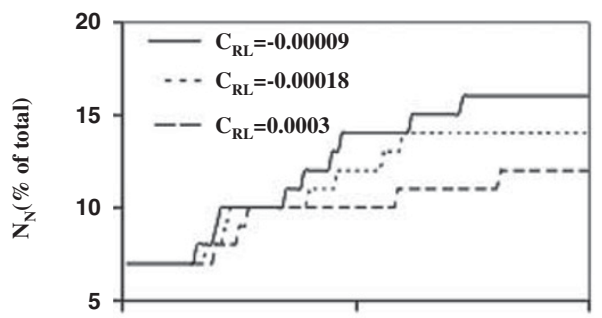

b

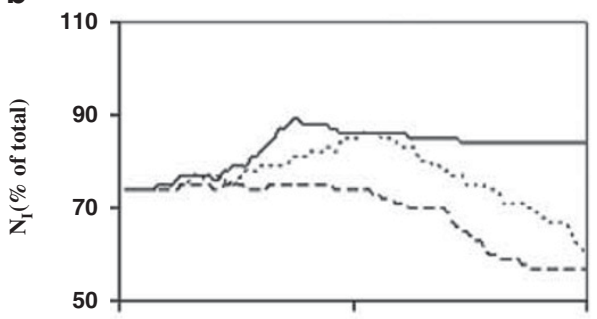

C

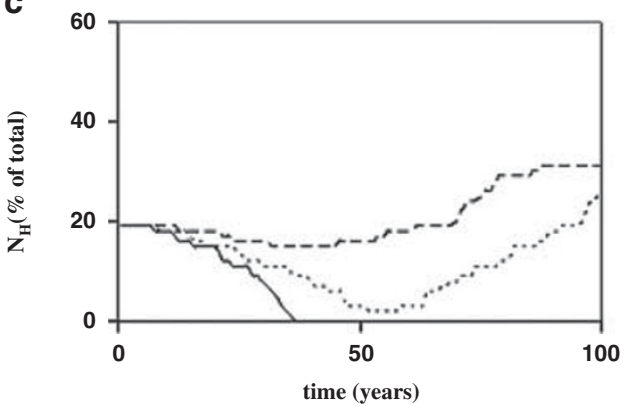

d

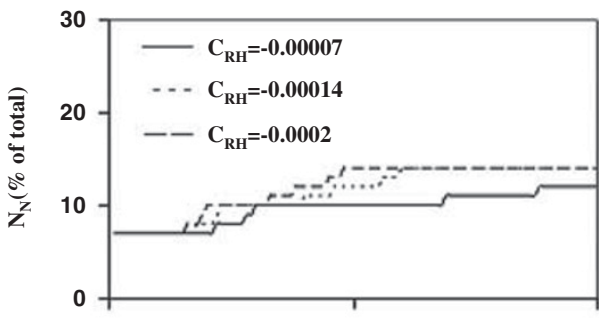

e

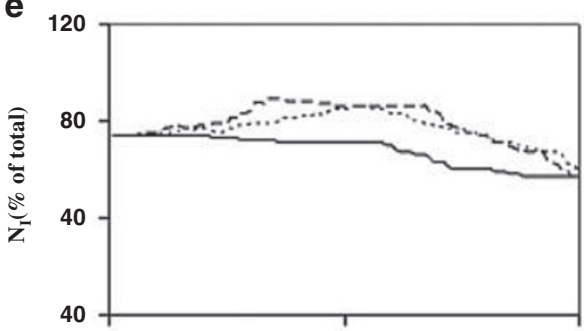

f

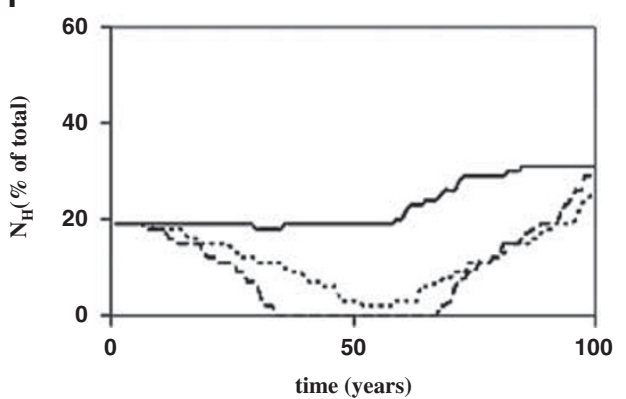

Figure 8 | Impact of differential response on institutional dynamics. The left panels represent results in terms of three values of differential response to low performers; the right panels represent results in terms of three values of differential response to high performers. The differential response has less effect on the number of non-performers than on the number of intermediate and high performers. The differential response provides an effective control variable for institutional performance.

(number of non-performers). In particular, certain projective designs could help to achieve sustained and improved performance.

The novel feature in our formalism is a somewhat mechanistic, and deterministic, description of time evolution of a research organization. A primary conclusion from our study is that a research organization may be quantitatively and consistently described as an initial-boundary value dynamical system. In the present case, the model demonstrates complex behaviour that a research institution can exhibit in response to internal as well as external factors. For example, if the individual performance exceeds the global benchmark $\left(x_{i}(t)>X_{G}\right)$, it creates a reduction; thus the term $X_{G}-x_{i}(t)$ becomes negative. A similar argument also applies to $X_{N}-x_{i}(t)$.

We have not carried out any case study at this stage; the initial fundamental purpose of this investigation has been to develop the model and study its behaviour. To carry out case studies for a given institution, it will be necessary to specify parameter $\alpha$, and use evolution of publication data in time (year). For an institutional structure where there is a single identifiable authority, the response parameter can be identical; otherwise, this parameter also needs to be specified. It is thus neither practical, nor intended, to claim the numbers are representative of any particular organization; the present work provides a generic formalism that can be applied to any given system. That said, empirical validation of such a model can be planned with data on individual members' performance, with reference to parameters that define the driving forces. Once these parameters are quantified based on a fraction of the members for certain years, the calibrated model can be used for validation using member performance and years not used in the process of calibration. The parameters will have to be calibrated using actual institutional data for application. While we have considered performance criteria in terms of number of SCI publication, the methodology is somewhat generic, as the criteria can be modified or extended without any change in the structure of the model (equations).

It is possible to consider a more complex version of the model by introducing phased recruitment based on dynamical selection criteria. Given the increasingly global nature of scientific research, it is necessary to consider both national and global benchmarks for performance. However, for most countries these two benchmarks are likely to be different, and driven by different dynamics. Another assumption in our model that can be relaxed is the constancy of the national and global benchmarks. It can be argued that as scientific research becomes more intensive, the average number of publications will rise at both at national and global level. However, the criteria for publications in SCI journals are also likely to become more stringent (higher rejection rates); thus the average performance may not change appreciably. Still, our assumptions of static national and global benchmarks thus have their limitations.

It could be argued that various organizational characteristics and policies would change sufficiently to render a 100-year, or 
a

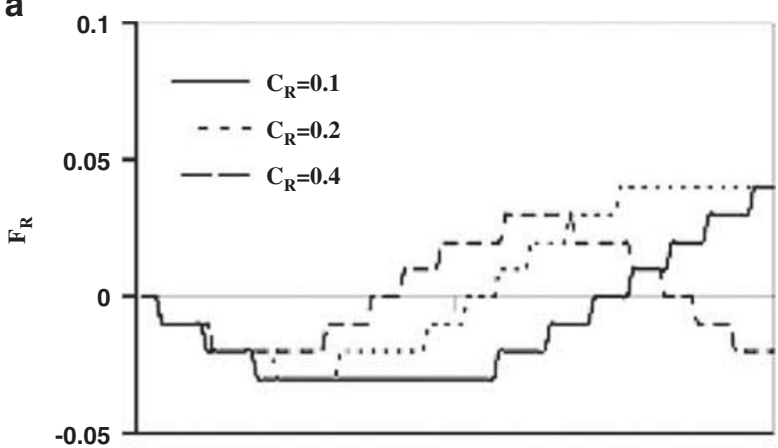

b

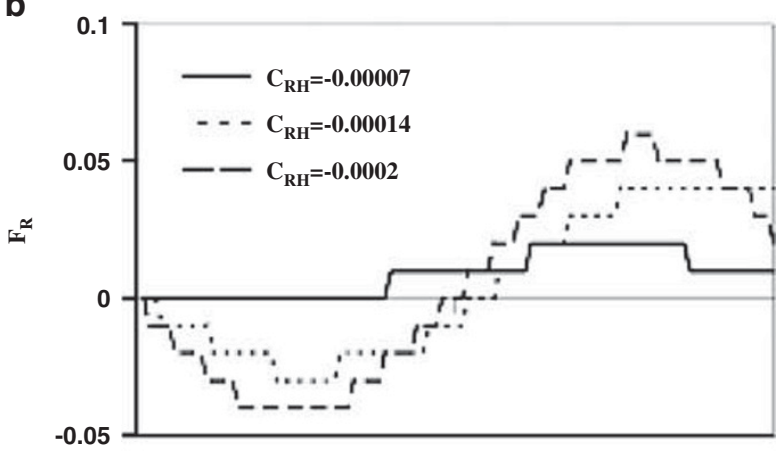

C

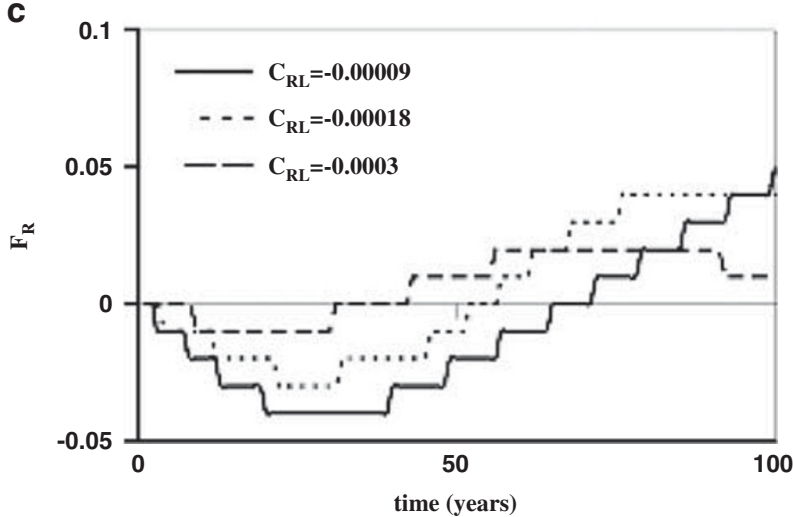

Figure 9 | Evolution of response of leadership for various strengths of response and coefficients of differential response to low and high performers. The leadership response itself can follow complex trajectories with characteristic variability as a part of the institutional dynamics.

even a 20-year, simulation meaningless. Leadership policy will have changed, and clearly no individual member will be active in an organization for 100 years. However, one reason for considering time-evolving leadership is to account for this kind of time dependence without reference to any particular member; what the formalism does not account for right now is disruptive situations (either large positive or negative influence). An implicit assumption in our formalism is that members leave the system after some years and are replaced with newcomers to maintain a constant total number. The formalism can be extended to a time varying total number; however, this is avoided for simplicity at this stage. Finally, because we are considering members' average performance, the entry or exit of individual members do not affect our conclusions.

The model can be easily modified to explore other social systems where individual performance (such as in a class room) is affected by collective performance, multiple benchmarks and peer response. However, it will be necessary to include additional processes, especially in an increasingly connected global network; these aspects should include other related processes like external drivers (Galam, 2004), global networking (Easley and Kleinberg, 2010) and even processes like judgment (Alicke et al., 2005). While these involve conceptual and methodological challenges, our work is expected to provide a basic framework.

\section{References}

Abelson R P (1964) Mathematical models of the distribution of attitudes under controversy. In: Fredericksen $\mathrm{N}$ and Gullicksen $\mathrm{H}$ (eds) Contributions to Mathematical Psychology. Holt, Rinehart \& Winston: New York, pp 142-160.

Alicke M D, Dunning D A and Krueger J I (2005) The Self in Social Judgment. Psychology Press: New York.

Allanach J, Tu H, Singh S, Willett P and Pattipati K (2004) Detecting, tracking, and counteracting terrorist networks via Hidden Markov Models. Paper presented at IEEE Aerospace Conference Proceedings, Big Sky, MT, IEEE (6-13 March).

Allport F H (1924) Social Psychology. Houghton Mifflin: Boston.

Antal T, Krapivsky P L and Redner S (2005) Dynamics of social balance on networks. Physical Review. E72: 036121

Baer M, Evans K, Oldham G R and Boasso A (2015) The social network side of individual innovation: A meta-analysis and path-analytic integration. Organizational Psychology Review; advance online publication 6 January, doi: $10.1177 / 2041386614564105$.

Barabasi A L, Jeong H, Neda Z, Ravasz E, Schubert A and Vicsek T (2002) Evolution of the social network of scientific collaborations. Physica A: Statistical Mechanics and Its Applications; 311 (3): 590-614.

Bardhan P (1989) The new institutional economics and development theory: A brief critical assessment. World Development; 17 (9): 1389-1395.

Bejan A and Merkx G W (2007) Construction Theory of Social Dynamics. Springer: New York, USA.

Bond R (2005) Group size and conformity. Group Processes Intergroup Relations; 8 (4): 331-354.

Brauer F and Castillo-Chavez C (2001) Mathematical Models in Population Biology and Epidemiology. Springer: New York.

Carrol T and Burton R M (2007) Organizations and complexity: Searching for the edge of chaos. CMOT; 6 (4): 319-337.

Castellano C, Fortunato S and Loreto V (2009) Statistical physics of social dynamics. Reviews of Modern Physics; 81 (2): 591-646.

Chavance B (2008) Institutional Economics. Routledge, Taylor \& Francis group: London.

Chen L, Gable G U and Hu H (2013) Communication and organizational social networks: A simulation model. CMOT; 19 (4): 460-479.

Commons J R (1934) Institutional Economics. Macmillan: New York.

Cronbach L J (1955) Processes affecting scores on understanding of others and assumed similarity. Psychological Bulletin; 52 (3): 177-193.

Easley D and Kleinberg J (2010) Networks, Crowds, and Markets: Reasoning about a Highly Connected World. Cambridge University Press: Cambridge.

Edelstein-Keshet L (2004) Mathematical Models in Biology. SIAM: Philadelphia, USA.

Facchetti G, Iacono G and Altafini C (2011) Computing global structural balance in large-scale signed social networks. Proceedings of the National Academy of Sciences; 108 (52): 20953-20958.

Farley J D (2007) Evolutionary dynamics of the insurgency in Iraq: A mathematical model of the battle for hearts and minds. Studies in Conflict and Terrorism; 30 (11): 947-962.

Fieldhouse E, Shryane N and Pickles A (2007) Strategic voting and constituency context: Modelling party preference and vote in multiparty elections. Political Geography; 26 (2): 159-178.

Flierl G, Grunbaum D, Levins S and Olson D (1999) From individuals to aggregations: The interplay between behavior and physics. Journal of Theoritical Biology; 196 (4): 397-454.

Friedman M and Friedman R (1984) The Tyranny of the Status Quo. Houghton Mifflin Harcourt: Boston.

Galam S (2004) The dynamics of minority opinions in democratic debate. Physica A; 336 (1): $56-62$.

Granovetter M (1985) Economic action and social structure: The problem of embeddedness. American Journal of Sociology; 91 (3): 481-510.

Gueron S, Levin S A and Rubenstein D I (1996) The dynamics of herds: From individuals to aggregations. Journal of Theoritical Biology; 182 (1): 85-98.

Harrison J R and Carrol G R (2002) The dynamics of cultural influence networks. CMOT; 8 (1): 5-30.

Hegselmann R and Flache A (1998) Understanding complex social dynamics: A plea for cellular automata based modelling. Journal of Artificial Societies and Social Simulation; 1 (3): 1. 
Heider F (1982) The Psychology of Interpersonal Relations. Psychology Press: New York. Hodgson G (1993) Economics and Evolution. Michigan University Press: Michigan, USA.

Hodgson G M (1998) The approach of institutional economics. Journal of Economic Literature; 36 (1): 166-192.

Hodgson G M (2000) What is the essence of Institutional economics. Journal of Economic Issues; 34 (2): 317-329.

Iacono G and Altafini C (2010) Monotonicity, frustration, and ordered response: An analysis of the energy landscape of perturbed large-scale biological networks. BMC Systems Biology; 4: 83.

Maanen J V and Barley S R (1984) Occupational communities: Culture and control in organizations. Research in Organizational behavior; 6: 287-365.

Matthies M, Malchow H and Kriz J (2001) Integrative Systems Approaches to Natural and Social Dynamics: System Science 2000. Springer-Verlog Heidelberg: New York.

McPherson J M, Popielarz P A and Drobnic S (1992) Social networks and organizational dynamics. American Sociological Review; 57 (2): 153-170.

Mogilner A, Edelstein-Keshet L, Bent L and Spiros A (2003) Mutual interactions, potentials, and individual distance in a social aggregation. Journal of Mathematical Biology; 47 (4): 353-389.

Nagel M (2010) A mathematical model of democratic elections. Current Research Journal of Social Sciences; 2 (4): 255-261.

Newcomb T M, Turner R H and Converse P E (1965) Social Psychology: The Study of Human Interaction. Holt Rinehart and Winston: New York.

Palla G, Barabsi A L and Vicsek T (2007) Quantifying social group evolution. Nature; 446 (7136): 664-667.

Rutherford M (2001) Institutional economics. Journal of Economic Perspectives; 15: 173-194.

Schotter A (2008) The Economic Theory of Social Institutions. Cambridge University Press: Cambridge.

Skyrms B and Pemantle R (2000) A dynamic model of social network formation. Proceedings of the National Academy of Sciences; 97 (16): 9340-9346.

Thalheim B (2012)The science and art of conceptual modelling In: Hameurlain A, Kung J and Wagner R (eds) Transactions on Large-Scale Data and KnowledgeCentres Systems VI. Special Issue on Database- and Expert-Systems Applications 7600pp 76-105.

Toral R and Tessone C J (2007) Finite size effects in the dynamics of opinion formation. Communication in Computational Physics; 2 (2): 177-195.

Tuma N B and Hannan M T (1984) Social Dynamics Models and Methods. Academic Press, INC: Florida.

Vugt M V and Schaller M (2008) Evolutionary approaches to group dynamics: An introduction. Group Dynamics: Theory, Research, and Practice; 12 (1): 1-6.
White D R, Owen-Smith J, Moody J and Powell W W (2004) Networks, fields and organizations: Micro-dynamics, scale and cohesive embeddings. CMOT; 10 (1): 95-117.

\section{Data Availability}

No Dataset were generated or analysed during the current study; all simulations can be generated based on the algorithms available in the published manuscript.

\section{Author Contributions}

All authors discussed the results and commented on the manuscript. PG formulated the algorithm, carried out literature review, interpreted the results and prepared the manuscript. DS and SN performed the simulations, carried out literature survey and PG and $\mathrm{SN}$ contributed to analysis and interpretation.

\section{Acknowledgements}

This work was supported by CSIR, India, under its Network Project Integrated Analysis for Impact, Mitigation and Sustainability. Note that Prashant Goswami's affiliation at the time of acceptance for publication was: Centre for Mathematical Modelling and Computer Simulation (repositioned CSIR Fourth Paradigm Institute), Bangalore, Karnataka, India

\section{Additional Information}

Competing interests: The authors declares no competing financial interests.

Reprints and permission information is available at http://www.palgrave-journals.com/ pal/authors/rights_and_permissions.html

How to cite this article: Goswami P, Nishad S N and Selvaratnam D (2015) A deterministic model of a research organization's evolution and dynamics of performance. Palgrave Communications 1:15012 doi: 10.1057/palcomms.2015.12.

\section{(c) (i)}

This work is licensed under a Creative Commons Attribution 3.0 International License. The images or other third party material in this article are included in the article's Creative Commons license, unless indicated otherwise in the credit line; if the material is not included under the Creative Commons license, users will need to obtain permission from the license holder to reproduce the material. To view a copy of this license, visit http://creativecommons.org/licenses/by/3.0/ 